\title{
Rhythm perception and differences in accent weights for musicians and nonmusicians
}

\author{
LLOYD A. DAWE \\ University of Western Ontario, London, Ontario, Canada \\ and \\ JOHN R. PLATT and RONALD J. RACINE \\ McMaster University, Hamilton, Ontario, Canada
}

\begin{abstract}
In order to investigate the contribution of harmonic-temporal and structural features to the perception of musical rhythm, three experiments were conducted in which a harmonic and a temporal accent were pitted against each other in such a way as to form five possible patterns. In three experiments, the temporal structure of various chord progressions was manipulated in an effort to determine the harmonic contributions to the inference of meter. The final experiment differed from the first two in the use of nondiatonic progressions that implied an unlikely key modulation. In all experiments, musicians and nonmusicians were requested to report perceived rhythm patterns in an attempt to determine the relative salience of various accents. Results indicated that changes in the temporal structure led to predictable change in an inferred meter, and that all diatonic chord progressions led to similar patterns of responses in which coincidences of harmonic, temporal, and metrical accents were perceptually salient events. Unusual progressions implying key modulations resulted in a qualitatively distinct pattern of results, and, in all experiments, amount of formal musical training was found to be a good predictor of the use of harmonic cues.
\end{abstract}

There have been many approaches to the study of rhythm. Of particular utility is the investigation of rhythmic structure through an analysis of how a musical sequence is temporally parsed on the basis of accents (Dawe, Platt, \& Racine, 1993; Jones, 1987). Accents have been identified with events that attract one's attention (Jones, 1987; Palmer \& Krumhansl, 1990), although use of the term has implied an even wider denotation that incorporates directed attentional energy (e.g., Clynes, 1982, 1986; Desain, 1992; Jones, 1987). As such, accents are both relative and dynamically functional in that they represent the perceptual salience of events. These variations in the proportional salience of events are captured through the notion of accent weights.

In keeping with Lerdahl and Jackendoff (1983), accents can be categorized into three types: phenomenal, structural, and metrical. A phenomenal accent is any event in the music that gives emphasis or stress to a moment (Lerdahl \& Jackendoff, 1983), resulting in a proportional capturing of a listener's attentional resources. Some examples of phenomenal accents are pitch jumps, chord changes, relatively long duration notes, and dynamics. Structural accents result from abstract cognitive princi-

This research was supported by a predoctoral fellowship from the Natural Sciences and Engineering Research Council of Canada to the first author and by Grant A8269 from the Natural Sciences and Engineering Research Council of Canada to the second author. Correspondence concerning this article should be addressed to L. A. Dawe, Department of Psychology, Social Science Centre, University of Western Ontario, London, ON, Canada N6A 5C2. ples associated with pitch and intervallic relationships within a given tonal context (Dawe et al., 1993; Lerdahl \& Jackendoff, 1983). An abundance of work on pitch relationships between various notes, chords, and tonal centers or keys (Bharucha \& Krumhansl, 1983; Cuddy, Cohen, \& Miller, 1979; Krumhansl, 1985, 1990; Krumhansl, Bharucha, \& Kessler, 1982; Krumhansl \& Castellano, 1983; Krumhansl \& Shepard, 1979) and intervallic relationships (Brown \& Butler, 1981; Butler, 1983, 1989) exists that may serve as a basis from which one may identify structural accenting; although this research has only recently been linked to accent identifications (Dawe et al., 1993). Finally, metrical accents arise from a periodic mental scheme (Lerdahl \& Jackendoff, 1983) that divides a musical sequence into time spans defined by the regular occurrence of strong and weak beats (Dawe et al., 1993; Lerdahl \& Jackendoff, 1983; Palmer \& Krumhansl, 1990). The strong and weak beats create a hierarchy of pulses that are equally spaced in time at any given level and that either double or triple in duration from one level in the hierarchy to the next (Lerdahl \& Jackendoff, 1983; Palmer \& Krumhansl, 1990). While metrical accents often coincide with phenomenal accents (Palmer \& Krumhansl, 1990), this is not a necessary condition (Dawe et al., 1993). The hierarchical nature of meter gives time points an identity independent of phenomenal or structural accents (Benjamin, 1984). This latter form of accenting is not a novel idea. In fact, several researchers have used the term accent to refer to targeted events of directed attention (Boltz, 1989; Jones, 1987), in part, because these events are perceived as if they were physically accented. They 
are, like phenomenal accents, better encoded and remembered than are events at other locations (Dowling, 1973; Jones, 1984, 1986).

Phenomenal and structural accents can create patterns of durations that serve as the basis for themes, motives, and phrases (i.e., grouping or phrase structure, Jones, 1987; Lerdahl \& Jackendoff, 1983). The phrase structure may depart from the rigidity of a metrical structure and, thus, contribute to the overall rhythm. Thus, an adequate delineation of rhythmic structure necessitates an investigation of phrase structure, metrical structure, and the relationship between the two. However, empirical studies of musical or rhythmic structure typically have concentrated on either phrase structure (Benjamin, 1984; Jones, 1981, 1982, 1984, 1987; Jones, Boltz, \& Kidd, 1982; Palmer \& Krumhansl, 1987a, 1987b) or metrical structure (Clarke, 1985; Palmer \& Krumhansl, 1990; Sloboda, 1983; Yeston, 1976), but rarely both. Occasionally, theorists will review and discuss the relationship between metrical and phrase structure (e.g., Berry, 1976; Cooper \& Meyer, 1960; Jones, 1990; Lerdahl \& Jackendoff, 1983; Palmer \& Krumhansl, 1990), but such reviews have more often than not been restricted to theoretical discussions and have not included perceptual research designed to investigate such interactions.

In addition, investigations of rhythm and, in particular, meter have often been restricted to the manipulation of temporal features (Essens \& Povel, 1985; Lee, 1985; Longuet-Higgins \& Lee, 1984; Povel, 1981; Povel \& Essens, 1985; Povel \& Okkerman, 1981). This research shows that metrical locations tend to be marked by temporally elongated notes (e.g., Clarke, 1985; Lee, 1985; Povel \& Okkerman, 1981), and that good phrases typically terminate with a relatively sustained tonic (e.g., Boltz, 1989; Vos, 1977). Other work has shown that long-duration notes are important for the perception and memory for phrases (Dowling, 1973). More recent research has included the contribution of melodic-based features to the parsing of a temporal sequence (Boltz, 1989; Jones, 1987, 1990, 1992; Jones \& Boltz, 1989; Palmer \& Krumhansl, 1987a, 1990).

In comparison, relatively little research has been directed toward the delineation of harmonic cues for rhythm (e.g., Dawe et al., 1993; Dawe, Platt, \& Racine, 1994; Palmer \& Krumhansl, 1987b; Smith \& Cuddy, 1989). This situation is all the more surprising given that many musical-theoretical views point to a role for harmonic factors in determining meter and rhythm (Cooper \& Meyer, 1960; Lerdahl \& Jackendoff, 1983).

This study was designed in an attempt to rectify this situation and to build on some initial studies done on the perceptual salience of harmonic accents in rhythm determination (Dawe et al., 1993, 1994). An earlier investigation (Dawe et al., 1994) of the contributions of various accents to the inference of a meter found that when different metrical structures supported by time spans defined by harmonic, melodic, and temporal accents are placed in conflict with one another, subjects will predominantly select the meter defined by harmonic events (e.g., chord changes). In a different investigation (Dawe et al., 1993) of meter and phrase contributions to rhythm perception, harmonic and temporal cues were pitted against one another in such a way as to form different rhythm patterns in which the perceived location of a long-duration note $(\mathrm{L}=444 \mathrm{msec})$ relative to four shortduration notes $(\mathrm{S}=222 \mathrm{msec})$ could vary on the basis of the location of a chord change. The stimulus patterns were iteratively presented, and subjects had to identify the perceived rhythm from among five alternatives (i.e., LSSSS, SLSSS, SSLSS, SSSLS, or SSSSL), while tapping to the beat of the presentation, in an effort to determine the inferred metrical structure. In keeping with the previous findings, harmonic factors were the principal determinant of the reported rhythm. In addition, the inference of metrical structure as determined by the pattern of tapping responses was critically tied to changes in the frequency of chord presentations (i.e., the composite rhythm) even though the timing between chord changes (i.e., the harmonic rhythm) was held constant. When the frequency of chord presentation was equal to the frequency of chord change (i.e., $1,332 \mathrm{msec}$ ), subjects inferred a $3 / 4$ meter. In contrast, when a chord was presented six times, each for a duration of $222 \mathrm{msec}$ before changing, tapping responses were indicative of a $6 / 8$ meter. As well, an interaction between rhythmic preferences and the inferred meter was found in that study. When the inferred meter was $6 / 8$ time, subjects showed a preference for harmonically defined rhythm patterns LSSSS and SSSLS; when the inferred meter was $3 / 4$ time, subjects showed a preference for the harmonically defined rhythm patterns of LSSSS, SSLSS, and SSSSL.

The purpose of this study was to expand upon these initial experiments and to investigate some alternative explanations for the reported effects (Dawe et al., 1993). Specifically, the motivation behind these experiments was threefold. First, in the previous study, each trial included a temporal sequence consisting of middle $\mathrm{C}$ and only one chord progression employed as a harmonic accompaniment (i.e., C-G-C triads in root position; see Table 1). This led to the possibility that structural factors could have contributed to the perceptual salience of the chord change, since the combination of the dominant chord and middle $\mathrm{C}$ would have resulted in a relatively dissonant suspension. To determine the extent to which this dissonance could have contributed to the results found in that study, different chord progressions were employed in these experiments that varied in the relative consonance of the constituent triadic notes and the middle $C$ of the temporal line. Second, the perceptual salience of temporal sequences that coincided with metrical accents in Dawe et al. (1993) could have been inflated due to the behavioral measure employed (i.e., tapping to the "beat" of the presentation). By having subjects give a behavioral response at specific locations in each sequence, a bias for certain rhythms could have been established that, in the absence of tapping, may not have existed. In order to investigate this possibility, the same composite and harmonic rhythms employed in Dawe et al. (1993) 
were used in this study, but subjects were asked only to identify the perceived rhythm in the absence of a tapping response. If the pattern of results is found to be the same as in Dawe et al. (1993), then this would support the interpretation that the increased salience of certain temporal sequences was due to the directing of attentional energies (i.e., metrical accents). Finally, we were interested in the effects of musical training on the relative weights assigned to different accents.

\section{EXPERIMENT 1}

In Experiment 1, three progressions were employed as a harmonic accompaniment to a temporal sequence. The composite and harmonic rhythmic features of this accompaniment should result in the inference of a $6 / 8$ meter and, on the basis of previous research, a bias for the rhythms of LSSSS and SSSLS (Dawe et al., 1993).

\section{Method}

Subjects. Eighteen volunteers participated in Experiment 1. The reported number of years of musical training ranged from 0 to 12 (see Figure 1a). The subjects, who were recruited from introductory psychology classes and given course credit, ranged from 18 to 22 years of age, with a median of 19.5 years.

Stimuli. Table 1 provides an example of the stimuli used in this experiment. The stimuli consisted of a temporal line in which a long-duration note ( $\mathrm{L}=444 \mathrm{msec}$ ) followed by four short-duration notes $(\mathrm{S}=222 \mathrm{msec}$ ) was presented 10 times (i.e., LSSSSLSSSS). The 50 notes presented were all $\mathrm{C}_{4}$ in order to eliminate melodic phenomenal and structural accents. On any given trial, 1 of 15 different harmonic accompaniments that consisted of simple diatonic triads in root position were presented with the temporal line. Three types of progression and five different chord-change locations were used. The three types of progression were $\mathrm{C}_{3}-\mathrm{G}_{3}-$ $\mathrm{C}_{3}, \mathrm{C}_{3}-\mathrm{F}_{3}-\mathrm{C}_{3}$, and $\mathrm{F}_{3}-\mathrm{G}_{3}-\mathrm{F}_{3}$. Each chord was presented six times (222 msec each) before changing, with each presentation lasting the same duration as a short-duration note in the temporal line. By systematically varying the location of the chord changes in relation to the long-duration tone, the melody was broken into five differ- ent rhythm patterns (i.e., LSSSS, SLSSS, SSLSS, SSSLS, and SSSSL). The stimuli consisted of an FM-synthesized piano timbre presented at approximately $72 \mathrm{~dB}$ SPL(B), with the temporal line being at a slightly higher level than the harmonic accompaniment (approximately 4- $\mathrm{dB}$ difference) to facilitate perception of the rhythm. The sequence was presented at a tempo of 135 beats per minute, if the quarter note received the beat, to ensure that the perceived tactus for either a $3 / 4$ or a $6 / 8$ meter would fall within the preferred range from 200 to $900 \mathrm{msec}$ (Fraisse, 1963; Handel, 1989).

Apparatus. The stimuli were generated by a Yamaha CX5M music computer and presented via a Yamaha CA-140 amplifier to Realistic Pro-2 earphones. Each subject sat in a sound-attenuated AEC chamber and responded to the stimuli by moving a computer mouse that controlled the movements of a cursor visible on a Hitachi 1481 color monitor situated at eye level directly in front of a glass window of the chamber.

Procedure. A unique random ordering of 60 trials (4 repetitions of the 15 stimuli) was presented to each subject. The subjects were instructed to listen to each trial and choose which rhythm pattern they had heard by placing a cursor, which they controlled with a joystick, into one of five labeled boxes (LSSSS, SLSSS, SSLSS, SSSLS, and SSSSL) visible on the monitor. The subjects were also told that there were no correct answers and that it was possible that all trials would sound as if they had identical rhythms. ${ }^{1}$ They were under no time constraints and started each trial by placing the cursor in a startbox, which was located an equal distance from each of the five response boxes, and then pressing a button. While each trial played, the cursor disappeared to prevent early responses and reappeared in the startbox upon the end of stimulus presentation. Prior to the experiment, the experimenter ensured that the subjects understood what each schemata represented by having them tap each of the five rhythms. Data were collected through a standard questionnaire on musical training, family musicians, general attitude toward music, age, and gender.

\section{Results and Discussion}

The first analysis was to identify possible effects of formal musical training. Response profiles consisting of percentage counts for the dependent variable of rhythmic choice (LSSSS, SLSSS, SSLSS, SSSLS, or SSSSL) in each of the three harmonic conditions were computed for each subject resulting in a $5 \times 15$ matrix. The 18 matri-
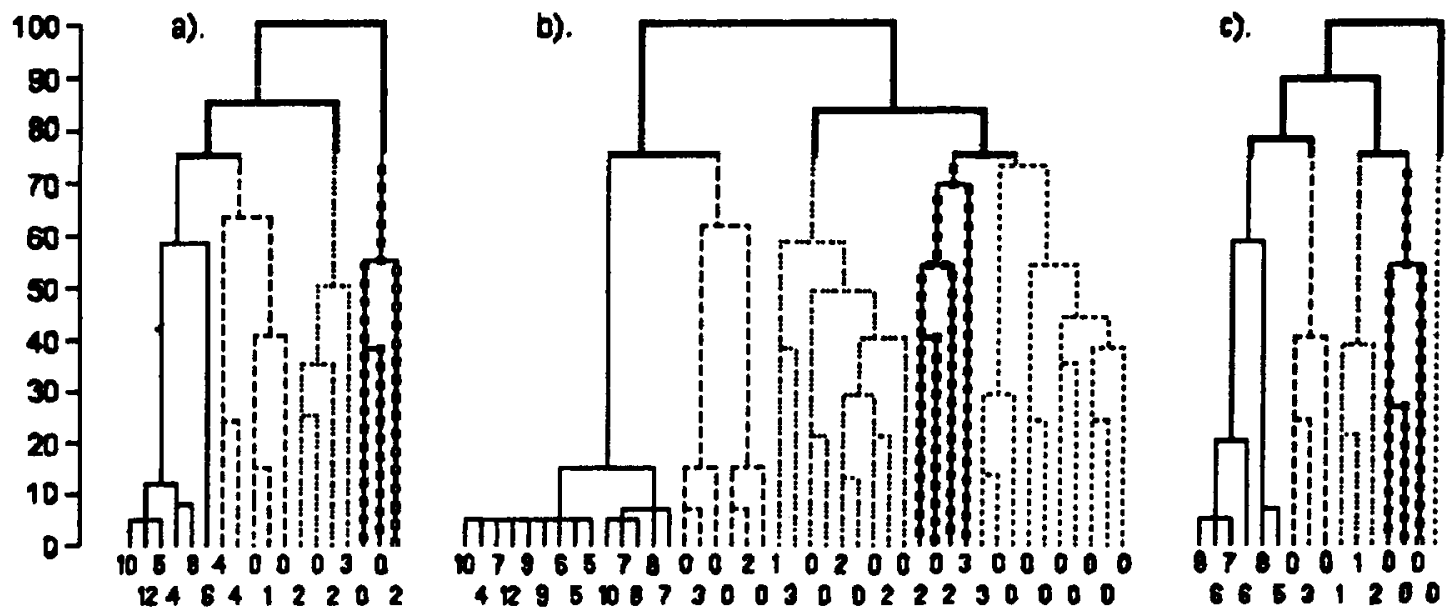

Figure 1. Hierarchical clustering (Ward's method) of subjects based on individual response profiles for Experiments 1 (a), 2 (b), and 3 (c). The reported number of years of musical training is given below each case. The ordinate represents a pmoportional measure of dissimilarity relative to the maximum Euclidean distance. 
Table 1

A Schematic Example of Stimuli Used in Experiments 1, 2, and 3

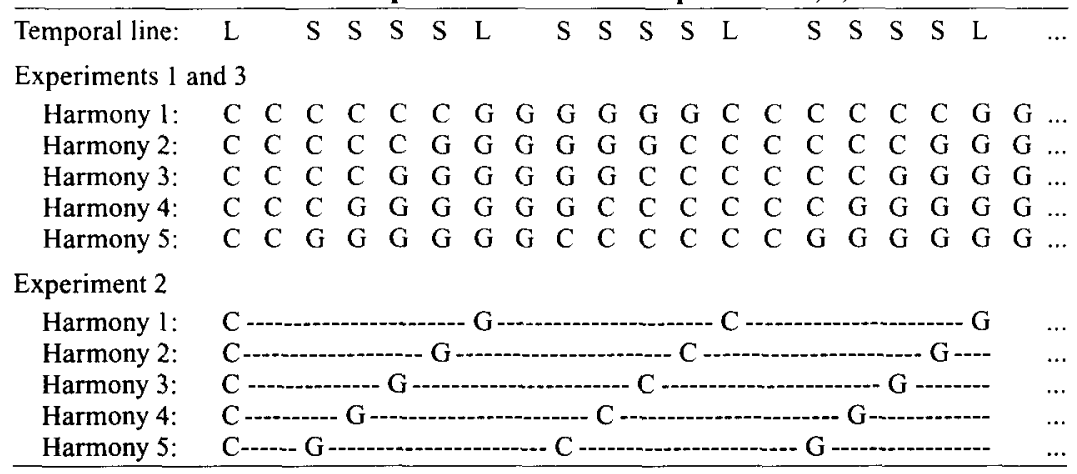

Note-This table is read by following the location of a chord change upward to see where it intersects the temporal line, which consisted of middle $\mathrm{C}$ being presented for either a long $(\mathrm{L})$ or a short (S) duration. The five harmonies create five rhythm patterns on the basis of a chord change: LSSSS, SLSSS, SSLSS, SSSLS, and SSSSL. Although only a C-G-C progression is shown in this table, the experiments employed other cadences.

ces (one for each subject) were then analyzed by means of hierarchical clustering. The solution is shown in Figure 1a, with the reported number of years of formal musical training beneath each case. Systematically conducted loglinear analyses that included and excluded all individual subjects from the different groups supported the identification of four distinct response groups shown in Figure 1a. The 6 subjects with the most number of years of formal training formed a cluster on the left-hand side of the solution, with the remaining subjects creating three other clusters.

Because the measures used in this experiment were discrete and categorical, loglinear analyses were performed on the data, with three design and one response variable. The design variables were cluster membership as determined by the hierarchical solution, the rhythm pattern as defined by the location of a chord change, and the type of chord progression. The response variable was choice of rhythm. An appropriate model was determined by first conducting tests of partial and marginal association (for a discussion of these tests, see Dawe et al., 1993). The results of the association tests indicate that the significant effects were cluster [partial and marginal, $\chi^{2}(3)=59.32, p<.001$ ], choice [partial and marginal, $\chi^{2}(4)=75.30, p<.001$ ], choice $\times$ cluster [partial, $\chi^{2}(12)=$ $138.37, p<.001$; marginal, $\chi^{2}(12)=124.77, p<.001$ ], choice $\times$ location [partial, $\chi^{2}(16)=308.32, p<.001$; marginal, $\left.\chi^{2}(16)=294.50, p<.001\right]$, and choice $\times$ location $\times$ cluster [partial, $\chi^{2}(48)=199.25, p<.001$; marginal, $\left.\chi^{2}(48)=195.70, p<.001\right]$. The second stage of the analysis involved the selection of a model that best fitted the observed data. This was done by automatic stepwise model selection (Dawe et al., 1993). The bestfitting model was choice $\times$ location $\times$ cluster $\left[\chi^{2}(200\right.$, $N=1,080)=161.05, p=.98]$.

The significant effect of choice and its interaction with the location of a chord change are illustrated in Table 2 . Two rhythm patterns were chosen significantly more often than chance: LSSSS and SSSLS. The inter- action of choice with location of chord change indicates that, overall, the subjects selected rhythm patterns on the basis of the location of a chord change. This is evident by noting the percentage of responses falling along the negative diagonal of the response profile (Table 2). This effect, however, must be interpreted with caution, since both the cluster analysis and the presence of higher order interactions suggest variability in the pattern of responses given across subjects.

Table 3 shows the different pattern of rhythmic choices as a function of cluster membership and location of the chord change. Subjects in Cluster 1, who reported the most amount of formal training, selected rhythm patterns primarily on the basis of the chord-change location. There was no evidence that any one rhythm pattern was selected more than others, although the rhythm patterns SLSSS and SSLSS were selected significantly less than chance. The pattern of responses for Cluster 1 indicates a preference for rhythm patterns defined by accent coincidences (Benjamin, 1984; Berry, 1976; Jones, 1987, 1992). This group selected LSSSS most when the pattern was supported by the coincidence of a chord-change accent, an inferred metrical accent (in either $6 / 8$ or $3 / 4$ time), and the accent associated with the onset of the relatively long duration note. ${ }^{2}$ The next two preferred rhythm

Table 2

Rhythm Choice and Choice $\times$

Chord-Change Location in Experiment 1

\begin{tabular}{cccccccc}
\hline & \multicolumn{2}{c}{ Rhythm on the Basis of the Chord Change } \\
\cline { 2 - 7 } Rhythmic Choice & LSSSS & SLSSS & SSLSS & SSSLS & SSSSL & Mean \\
\hline LSSSS & $56.9^{*}$ & 22.2 & 22.2 & $17.1 \dagger$ & 19.4 & $27.6^{*}$ \\
SLSSS & $7.4 \dagger$ & $28.2^{*}$ & $9.7 \dagger$ & $6.9 \dagger$ & $10.2 \dagger$ & $12.5 \dagger$ \\
SSLSS & $6.9 \dagger$ & 20.4 & $34.3^{*}$ & $8.8 \dagger$ & $5.6 \dagger$ & $15.2 \dagger$ \\
SSSLS & $11.6 \dagger$ & $17.1 \dagger$ & 25.0 & $48.1^{*}$ & 19.9 & $24.4^{*}$ \\
SSSSL & $17.1 \dagger$ & $12.0 \dagger$ & $8.8 \dagger$ & 19.0 & $44.9^{*}$ & 20.4 \\
$N$ & 216 & 216 & 216 & 216 & 216 & 1080 \\
$M S_{\mathrm{e}}$ & 2.7 & 2.7 & 2.7 & 2.7 & 2.7 & 1.2 \\
\hline Note-Table entries are percents. & *Higher than expected & † tower
\end{tabular}

than expected $(p<.025)$. 
Table 3

Rhythm Choice $\times$ Chord-Change Location and Cluster Membership in Experiment 1

\begin{tabular}{|c|c|c|c|c|c|c|}
\hline \multirow[b]{2}{*}{ Rhythmic Choice } & \multicolumn{5}{|c|}{ Rhythm on the Basis of the Chord Change } & \multirow[b]{2}{*}{ Mean } \\
\hline & LSSSS & SLSSS & SSLSS & SSSLS & SSSSL & \\
\hline \multicolumn{7}{|c|}{ Cluster 1} \\
\hline LSSSS & $79.2^{*}$ & 12.5 & $8.3 \div$ & $2.8 \dagger$ & $5.6 \dagger$ & 21.7 \\
\hline SLSSS & $2.8 \dagger$ & $54.2 *$ & 11.1 & $4.2 \dagger$ & $6.9 \dagger$ & $15.8 \dagger$ \\
\hline SSLSS & $2.8^{\dagger}$ & 16.7 & $52.8^{*}$ & $4.2 \dagger$ & $-\dagger$ & $15.3 \dagger$ \\
\hline SSSLS & $6.9 \dagger$ & $8.3 \dagger$ & 20.8 & $69.4^{*}$ & 12.5 & 23.6 \\
\hline SSSSL & $8.3 \dagger$ & $8.3 \dagger$ & $6.9 \dagger$ & 19.4 & $75.0^{*}$ & 23.6 \\
\hline$N$ & 72 & 72 & 72 & 72 & 72 & 360 \\
\hline$M S_{\mathrm{e}}$ & 4.7 & 4.7 & 4.7 & 4.7 & 4.7 & 2.1 \\
\hline \multicolumn{7}{|c|}{ Cluster 2} \\
\hline LSSSS & $53.3^{*}$ & 10.0 & 21.7 & 16.7 & 15.0 & 23.3 \\
\hline SLSSS & 10.0 & 18.3 & 15.0 & $6.7 \dagger$ & 13.3 & $12.7^{\dagger}$ \\
\hline SSLSS & 10.0 & $43.3^{*}$ & 18.3 & 18.3 & $5.0^{\dagger}$ & 19.0 \\
\hline SSSLS & 10.0 & 16.7 & $35.0^{*}$ & $38.3^{*}$ & 23.3 & $24.7^{*}$ \\
\hline SSSSL & 16.7 & 11.7 & 10.0 & 20.0 & $43.3^{*}$ & 20.3 \\
\hline$N$ & 60 & 60 & 60 & 60 & 60 & 300 \\
\hline$M S_{\mathrm{e}}$ & 5.2 & 5.2 & 5.2 & 5.2 & 5.2 & 2.3 \\
\hline \multicolumn{7}{|c|}{ Cluster 3} \\
\hline LSSSS & 22.9 & 12.5 & 10.4 & 10.4 & 20.8 & 15.4 \\
\hline SLSSS & 10.4 & 22.9 & $\epsilon^{\dagger}$ & 10.4 & $8.3 \dagger$ & $10.4 \dagger$ \\
\hline SSLSS & $4.2 \uparrow$ & $8.3 \dagger$ & $43.8^{*}$ & $4.2 \dagger$ & 14.6 & 15.0 \\
\hline SSSLS & 29.2 & $39.6^{*}$ & $33.3^{*}$ & $52.1^{*}$ & $33.3^{*}$ & $37.5^{*}$ \\
\hline SSSSL & $33.3^{*}$ & 16.7 & 12.5 & 22.9 & 22.9 & 21.7 \\
\hline$N$ & 48 & 48 & 48 & 48 & 48 & 240 \\
\hline$M S_{\mathrm{e}}$ & 5.8 & 5.8 & 5.8 & 5.8 & 5.8 & 2.6 \\
\hline \multicolumn{7}{|c|}{ Cluster 4} \\
\hline LSSSS & $63.9^{*}$ & $75.0^{*}$ & $66.7^{*}$ & $55.6^{*}$ & $52.8^{*}$ & $62.8^{*}$ \\
\hline SLSSS & $8.3 \dagger$ & $-\dagger$ & 11.1 & $8.3 \dagger$ & 13.9 & $8.3 \dagger$ \\
\hline SSLSS & 13.9 & $5.6 \dagger$ & 11.1 & $8.3 \dagger$ & $5.6 \dagger$ & $8.9+$ \\
\hline SSSLS & $-\dagger$ & $5.6 \dagger$ & $5.6 \dagger$ & 16.7 & 11.1 & $7.8 \dagger$ \\
\hline SSSSL & 13.9 & 13.9 & $5.6 \dagger$ & 11.1 & 16.7 & $12.2 \dagger$ \\
\hline$N$ & 36 & 36 & 36 & 36 & 36 & 180 \\
\hline$M S_{\mathrm{e}}$ & 6.7 & 6.7 & 6.7 & 6.7 & 6.7 & 3.0 \\
\hline
\end{tabular}

Note-Table entries are percents. ${ }^{*}$ Higher than expected. †Lower than expected $(p<.025)$.

patterns were SSSSL and SSSLS when they were each supported by the coincidence of two accents. The rhythm of SSSSL was selected when its onset was supported by a chord change and the termination of the relatively long duration note. The rhythm of SSSLS was selected when its onset was defined by the chord-change location and what we believe is an inferred metrical accent for a $6 / 8$ meter.

The subjects in Cluster 2 similarly showed a preference for the rhythm patterns defined by accent coincidence, but they qualitatively differed from the first cluster in two important ways. They showed no evidence of selecting rhythm patterns on the basis of the chord-change location alone (e.g., when the chord-change-based rhythm pattern was SLSSS, the subjects selected the rhythm SSLSS most often), and there was a bias for the rhythm pattern of SSSLS, which was selected significantly more often than chance. The subjects in Clusters 3 and 4 also displayed biases but for the rhythm patterns of SSSLS and LSSSS, respectively, regardless of the rhythm pattern supported by the chord-change position.
The bias for rhythm patterns LSSSS or SSSLS observed in this experiment is meaningful. Even those clusters that did not show an overall bias for any given rhythm pattern showed some preference for these two patterns when they were defined by a chord-change location. We believe these two specific rhythms were preferentially selected because a 6/8 meter was inferred. Such an interpretation is consistent with Dawe et al. (1993), in which the use of the same type of harmony led to the inference of a $6 / 8$ meter and a bias for rhythms LSSSS and SSSLS.

It is noteworthy that the type of chord progression did not enter into any significant effects. An examination of the percentage count tables for each chord progression showed that the pattern of results reported in Table 2 is representative of all three chord progressions.

\section{EXPERIMENT 2}

In Experiment 2, the same progressions were employed except the composite and harmonic rhythms were changed to result in the inference of a 3/4 meter, and a bias for the rhythms LSSSS, SSLSS, and SSSSL (Dawe et al., 1993).

\section{Method}

Subjects. Forty-three experimentally naive volunteers participated in this experiment. The subjects ranged in age from 18 to 28 (median $=19$ years) and were recruited from introductory psychology classes as part of course requirements.

Stimuli. The stimuli were similar to those used in Experiment 1, except each chord of the harmonic accompaniment was presented only once for a duration of 3 beats in common time $(1,332 \mathrm{msec})$ before changing (see Table 1).

Apparatus and Procedure. The procedure and equipment were the same as those employed in Experiment 1.

\section{Results and Discussion}

Response profiles for each subject were analyzed by means of hierarchical clustering. The solution, as shown in Figure $1 b$ (with the number of years of formal training beneath each case), consisted of five groups as determined by loglinear analyses. The first group (shown in Figure $1 \mathrm{~b}$ by the solid lines) consisted of a relatively tight cluster of 14 subjects with the most number of years of formal musical training. The other four clusters, which consisted of subjects with less than 4 years of training, showed greater variability in their response patterns, indicated in the figure by relatively high connections (greater Euclidian distance) among the group members.

A loglinear analysis was performed on the data using the same design and response variables from Experiment 1 . The results of the tests for partial and marginal association indicate that the significant effects were choice [partial and marginal, $\chi^{2}(4)=13.19, p<.01$ ], cluster [partial and marginal, $\chi^{2}(1)=12.21, p<.001$ ], choice $\times$ cluster [partial, $\chi^{2}(4)=21.50, p<.001 ;$ marginal, $\chi^{2}(4)=$ $17.23, p<.01]$, choice $\times$ location [partial, $\chi^{2}(16)=$ $1,216.70, p<.001$; marginal, $\left.\chi^{2}(16)=1,212.08, p<.001\right]$, and choice $\times$ location $\times$ cluster [partial, $\chi^{2}(16)=776.01$, $p<.001$; marginal, $\left.\chi^{2}(16)=774.34, p<.001\right]$. The auto- 
matic stepwise model selection indicates that the bestfitting model was choice $\times$ location $\times$ cluster $\left[\chi^{2}(100\right.$, $N=2,580)=96.74, p=.57]$. Because this interaction contains all of the significant lower order effects, the results can be completely specified by examining this interaction.

Table 4 shows the different pattern of rhythmic choices as a function of cluster membership and location of the chord change. Overall, the percentage of responses for rhythm patterns defined by a chord-change location was higher in Experiment 2 than in Experiment 1. This was undoubtedly due to the associated temporal accent in the harmonic accompaniment. The subjects in Cluster 1 selected rhythms primarily on the basis of the chord-

Table 4

Rhythm Choice $\times$ Cluster Membership and Its Interaction With Chord-Change Location in Experiment 2

\begin{tabular}{ll}
\hline & Rhythm on the Basis of the Chord Change \\
\cline { 2 - 2 } Rhythmic Choice LSSSS SLSSS SSLSS SSSLS SSSSL Mean
\end{tabular}
Rhythmic Choice LSSSS SLSSS SSLSS SSSLS SSSSL Mean

\begin{tabular}{|c|c|c|c|c|c|c|}
\hline \multicolumn{7}{|c|}{ Cluster 1} \\
\hline LSSSS & $95.2^{*}$ & $6.5 \dagger$ & - & $1.8 \dagger$ & $3.0 \dagger$ & 21.3 \\
\hline SLSSS & $1.2 \dagger$ & $80.4^{*}$ & $3.6 \dagger$ & $3.6 \dagger$ & $1.8 \dagger$ & 18.1 \\
\hline SSLSS & $-\dagger$ & $6.0 \dagger$ & $90.5^{*}$ & $10.1 \dagger$ & $1.2 \dagger$ & 21.6 \\
\hline SSSLS & $1.2 \dagger$ & $5.4 \dagger$ & $5.4 \dagger$ & $82.1^{*}$ & $5.4 \dagger$ & 19.9 \\
\hline SSSSL & $2.4 \dagger$ & $1.8 \dagger$ & $0.6 \dagger$ & $2.4 \dagger$ & $88.7^{*}$ & 19.2 \\
\hline$N$ & 168 & 168 & 168 & 168 & 168 & 840 \\
\hline$M S_{\mathrm{c}}$ & 3.1 & 3.1 & 3.1 & 3.1 & 3.1 & 1.4 \\
\hline \multicolumn{7}{|c|}{ Cluster 2} \\
\hline LSSSS & $55.6^{*}$ & 15.3 & $2.8 \dagger$ & 11.1 & $8.3 \dagger$ & 18.6 \\
\hline SLSSS & $6.9 t$ & $38.9^{*}$ & 11.1 & $8.3 \dagger$ & 13.9 & $15.8 \dagger$ \\
\hline SSLSS & 18.1 & 25.0 & $56.9^{*}$ & 18.1 & $6.9+$ & $25.0^{*}$ \\
\hline SSSLS & 12.5 & 15.3 & 25.0 & $54.2 *$ & 11.1 & 23.6 \\
\hline SSSSL & $6.9 \dagger$ & $5.6 \dagger$ & $4.2 \dagger$ & $8.3 \dagger$ & $59.7^{*}$ & 16.9 \\
\hline$N$ & 72 & 72 & 72 & 72 & 72 & 360 \\
\hline$M S_{\mathrm{e}}$ & 4.7 & 4.7 & 4.7 & 4.7 & 4.7 & 2.1 \\
\hline \multicolumn{7}{|c|}{ Cluster 3} \\
\hline LSSSS & $66.7^{*}$ & 26.9 & 26.9 & 19.4 & $28.7^{*}$ & $33.7^{*}$ \\
\hline SLSSS & $9.3 \dagger$ & 18.5 & 15.7 & 26.9 & $39.8^{*}$ & 22.0 \\
\hline SSLSS & $12.0 \dagger$ & 22.2 & $29.6^{*}$ & 22.2 & 14.8 & 20.2 \\
\hline SSSLS & $2.8 \dagger$ & 20.4 & 15.7 & 16.7 & $7.4 \uparrow$ & $12.6 \dagger$ \\
\hline SSSSL & $9.3 \dagger$ & $12.0 \dagger$ & $12.0 \dagger$ & 14.8 & $9.3 \dagger$ & $11.5 \dagger$ \\
\hline$N$ & 108 & 108 & 108 & 108 & 108 & 540 \\
\hline$M S_{\mathrm{e}}$ & 3.8 & 3.8 & 3.8 & 3.8 & 3.8 & 1.7 \\
\hline \multicolumn{7}{|c|}{ Cluster 4} \\
\hline LSSSS & $47.9^{*}$ & 10.4 & 20.8 & 18.8 & 14.6 & 22.5 \\
\hline SLSSS & $-\dagger$ & 16.7 & $6.3 \dagger$ & 18.8 & 20.8 & $12.5 \dagger$ \\
\hline SSLSS & $6.3 \dagger$ & $6.3 \dagger$ & 10.4 & $2.1 \dagger$ & $2.1 \dagger$ & $5.4 \dagger$ \\
\hline SSSLS & 10.4 & 20.8 & 25.0 & 12.5 & $2.1 \dagger$ & $14.2 \dagger$ \\
\hline SSSSL & $35.4^{*}$ & $45.8^{*}$ & $37.5^{*}$ & $47.9^{*}$ & $60.4^{*}$ & $45.4^{*}$ \\
\hline$N$ & 48 & 48 & 48 & 48 & 48 & 240 \\
\hline$M S_{\mathrm{e}}$ & 5.8 & 5.8 & 5.8 & 5.8 & 5.8 & 2.6 \\
\hline \multicolumn{7}{|c|}{ Cluster 5} \\
\hline LSSSS & $27.5^{*}$ & 13.3 & 13.3 & 13.3 & 15.0 & $16.5^{\dagger}$ \\
\hline SLSSS & 14.2 & $9.2 \dagger$ & 14.2 & 20.0 & $33.3^{*}$ & 18.2 \\
\hline SSLSS & 16.7 & 25.0 & $35.0^{*}$ & 25.0 & 15.8 & $23.5^{*}$ \\
\hline SSSLS & 24.2 & 25.8 & 20.8 & 18.3 & 16.7 & 21.2 \\
\hline SSSSL & 17.5 & 26.7 & 16.7 & 23.3 & 19.2 & 20.7 \\
\hline$N$ & 120 & 120 & 120 & 120 & 120 & 600 \\
\hline$M S_{\mathrm{e}}$ & 3.7 & 3.7 & 3.7 & 3.7 & 3.7 & 1.6 \\
\hline
\end{tabular}

Note-Table entries are percents. *Higher than expected. $\dagger$ Lower than expected $(p<.025)$. change location. As in Experiment 1, the pattern of responses for Cluster 1 also supported the view that the subjects select rhythms defined by accent coincidences. Preferences were evident for rhythms LSSSS, SSLSS, and SSSSL when they were each supported by a chordchange accent. We interpret this pattern of responses as support for an inferred $3 / 4$ meter.

The subjects in Cluster 2 similarly showed a preference for the rhythm patterns defined by chord-change position and accent coincidence. Apart from the reported training differences, the members of this cluster were, compared with the first cluster, more variable in their responses, as indicated by the lower percentage counts along the negative diagonal in Table 4 and the higher levels of connections in Figure $1 \mathrm{~b}$. These subjects also showed a bias for the rhythm SSLSS.

The subjects in the final three clusters showed evidence of selecting the rhythm pattern LSSSS significantly more often than chance when it was supported by a chord change. The subjects in Clusters 3 and 5 also preferred the rhythm pattern of SSLSS when it was supported by a chord change. Strangely, the subjects in both Cluster 3 and Cluster 5 showed a preference for the rhythm SLSSS when the chord-change position defined a rhythm of SSSSL. It may be that the subjects in these two groups heard the stimuli under such conditions in a 6/8 meter. Such ambiguity regarding an inferred meter is not uncommon and has been previously noted among nonmusicians (Dawe et al., 1994).

Overall, the members of Clusters 3, 4, and 5 differed in their biases for selecting rhythm patterns LSSSS, SSSSL, and SSLSS, respectively. The presence of biases for these three rhythm patterns suggests that the harmonic presentation is somehow responsible since it is the only variable to have changed between the first and second experiments. The results are consistent with Dawe et al. (1993), in which the same harmonic presentations led to the inference of a $3 / 4$ meter and a bias for these three rhythm patterns.

As in Experiment 1, the type of chord progression did not enter into any significant effects, and an examination of the percentage count tables for each progression showed that the pattern of results reported in Table 4 was representative.

\section{EXPERIMENT 3}

It is somewhat surprising that in both Experiment 1 and Experiment 2, there were no significant differences found in the salience of chord changes as a function of chord progression. It may be that the structural accenting of a chord change does not affect the salience of the phenomenal event itself. Alternatively, it may be that the three cadence conditions that were used in the first two experiments were not sufficiently different. To test this possibility, Experiment 3 was conducted, using an unusual and dissonant chord progression. The harmonic and composite rhythmic features of Experiment 1 were also employed in Experiment 3 to see if the pattern of results from Experiment 1 could be replicated. 


\section{Method}

Subjects. Sixteen experimentally naive volunteers recruited from introductory psychology classes and given credit for participating served as subjects. Their ages ranged from 19 to 41 (median $=19$ years)

Stimuli. The stimuli in this experiment were the same as those used in Experiment 1 except that the progressions differed. The three types of harmonic accompaniment were $C_{3}-G_{3}-C_{3}, G_{3}-B_{3}$ dim$\mathrm{G}_{3}$, and $\mathrm{C} \#_{3}-\mathrm{B}_{3} \mathrm{dim}-\mathrm{C}_{3}$, with the triads in root position. As in Experiment 1 , each chord was presented six times before changing.

Apparatus and Procedure. The procedure and equipment were the same as in the previous experiments.

\section{Results and Discussion}

The hierarchical clustering solution of response profiles was similar to that found for the previous experiments. There were five distinct clusters (see Figure 1c). The first group consisted of the 6 musicians with the most number of years of formal musical training. Joining to this cluster were three groups of 3 subjects each. The final cluster consisted of 1 subject.

A loglinear analysis performed on the data with three design (cluster membership, location of a chord change, and type of chord progression) and one response (choice of rhythm) variable found the same significant effects as those reported in Experiments 1 and 2, with the addition of two interactions with the type of chord progression (choice $\times$ type of progression, and choice $\times$ location of a chord change $X$ type of progression). Post hoc loglinear analyses involving all possible pairwise comparisons of the types of chord progressions indicated that $\mathrm{re}^{\mathrm{eng}}$ sponse profiles for the chord progressions $C_{3}-G_{3}-C_{3}$ and $G_{3}-B_{3} \operatorname{dim}-G_{3}$ did not differ from one another but that each differed from the profiles for the chord progression $\mathrm{C}_{3}-\mathrm{B}_{3} \mathrm{dim}-\mathrm{C} \#_{3}$. Thus, for the purpose of fitting a model, all responses for the chord progressions $\mathrm{C}_{3}-\mathrm{G}_{3}-\mathrm{C}_{3}$ and $\mathrm{G}_{3}-\mathrm{B}_{3} \mathrm{dim}-\mathrm{G}_{3}$ were combined and contrasted with responses for $C \#_{3}-B_{3}$ dim- $C \#_{3}$. The design variables for this model included the cluster within which each subject fell, the rhythm pattern as defined by the location of a chord change, and the two groups of chord progressions (diatonic vs. nondiatonic progressions). The response variable was choice of rhythm.

The tests of partial and marginal association indicated the significant effects were choice [partial and marginal, $X^{2}(4)=13.55, p<.01$ ], cluster [partial and marginal, $\left.\chi^{2}(4)=212.17, p<.001\right]$, chord group [partial and marginal, $\chi^{2}(1)=95.79, p<.001$ ], choice $\times$ cluster [partial, $\chi^{2}(16)=140.19, p<.001$; marginal, $\chi^{2}(16)=130.98, p<$ .001 ], choice $\times$ chord group [partial, $\chi^{2}(4)=13.79, p<$ .05 ; marginal, $\left.\chi^{2}(4)=10.82, p<.05\right]$, choice $\times$ location $X$ cluster [partial, $\chi^{2}(64)=190.42, p<.001 ;$ marginal, $\left.\chi^{2}(64)=192.76, p<.001\right]$, and choice $\times$ location $X$ chord group [partial, $\chi^{2}(16)=33.32, p<.01$; marginal, $\left.\chi^{2}(16)=35.12, p<.01\right]$. The automatic stepwise model selection indicated a model that was almost a perfect fit. It was based on two three-way interactions of choice $\times$ location $\times$ chord group and choice $\times$ location $\times$ cluster $\left[\chi^{2}(100, N=960)=41.53, p=.995\right]$. The results can be completely specified by examining these two interactions.
Table 5 shows the response profiles of rhythmic choice $\times$ location of a chord change for the two chord groups. When the chord progression was diatonic, most choices were based on the location of the chord change as indicated by the large number of responses along the negative diagonal. Overall, there was a significant bias for the rhythm pattern LSSSS for these two progressions, and the percentage of responses for the rhythm pattern SSSLS approached significance. ${ }^{3}$ The rhythm patterns LSSSS and SSSLS were also given preference when the chord changes supported these rhythm patterns.

In contrast, the chord progression $\mathrm{C}_{3}-\mathrm{B}_{3} \mathrm{dim}-\mathrm{C} \#_{3}$ resulted in a profile in which there was no evidence of rhythm pattern biases. Responses for the rhythm patterns of SLSSS, SSLSS, SSSLS, and SSSSL were highest when these patterns were defined by a chord change, but there was no evidence of preferred rhythms based on accent coincidences or of an inferred metrical structure. Also, when the chord-change accent defined the rhythm LSSSS, the subjects reported hearing SLSSS. Analysis of individual response profiles indicated that every subject, except one (Cluster 5), showed this pattern of responding

Table 6 shows the effect of rhythmic choice $\times$ location of the chord change $\times$ cluster membership. The 6 musicians who constituted Cluster 1 gave the characteristic pattern of responses based on the location of the chord change. Most rhythmic responses occur along the negative diagonal of the response profile, with no clear prefrence for any specific rhythm pattern. The 3 subjects within Cluster 2 also showed a preference for rhythm patterns based on chord changes but were more variable in their responses than were members of Cluster 1. Members of Cluster 2 showed an overall bias for the rhythm pattern SSSLS. Members of Cluster 3 displayed profiles similar to those found for all subjects but one in response

Table 5

Rhythm Choice $\times$ Chord Progression Group and Its Interaction With Chord-Change Location in Experiment 3

Rhythm on the Basis of the Chord Change Rhythmic Choice LSSSS SLSSS SSLSS SSSLS SSSSL Mean

C-G-C and G-Bdim-G Progressions

\begin{tabular}{ccccccl} 
LSSSS & $53.9^{*}$ & 21.9 & 18.0 & 16.4 & 20.3 & $26.1^{*}$ \\
SLSSS & $11.7 \dagger$ & $39.8^{*}$ & 14.1 & 14.8 & $7.0 \dagger$ & 17.5 \\
SSLSS & $7 . \dagger^{\dagger}$ & $8.6 \dagger$ & $35.9^{*}$ & $12.5 \dagger$ & $12.5 \dagger$ & $15.3 \dagger$ \\
SSSLS & $10.2 \dagger$ & 16.4 & 21.1 & $44.5^{*}$ & 20.3 & 22.5 \\
SSSSL & 17.2 & 13.3 & $10.9 \dagger$ & $11.7 \dagger$ & $39.8^{*}$ & 18.6 \\
$N$ & 128 & 128 & 128 & 128 & 128 & 640 \\
$M S_{\mathrm{e}}$ & 3.5 & 3.5 & 3.5 & 3.5 & 3.5 & 1.6 \\
\multicolumn{7}{c}{} \\
\multicolumn{7}{c}{ C\#-Bdim-C\# Progression } \\
LSSSS & 18.8 & 14.1 & 21.9 & 17.2 & 21.9 & 18.8 \\
SLSSS & $42.2^{*}$ & $45.3^{*}$ & $7.8 \dagger$ & 10.9 & 12.5 & 23.7 \\
SSLSS & 18.8 & 14.1 & $42.2^{*}$ & 15.6 & $6.3 \dagger$ & 19.4 \\
SSSLS & 12.5 & $7.8 \dagger$ & 20.3 & $42.2^{*}$ & 21.9 & 20.9 \\
SSSSL & $7.8 \dagger$ & 18.8 & $7.8 \dagger$ & 14.1 & $37.5^{*}$ & 17.2 \\
$N$ & 64 & 64 & 64 & 64 & 64 & 320 \\
$M S_{\mathrm{e}}$ & 5.0 & 5.0 & 5.0 & 5.0 & 5.0 & 2.2 \\
\hline
\end{tabular}

Note-Table entries are percents. ${ }^{*}$ Higher than expected. $\quad$ Lower than expected $(p<.025)$. 
Table 6

Rhythm Choice $\times$ Cluster Membership and Its Interaction With Chord-Change Location in Experiment 3

Rhythm on the Basis of the Chord Change

Rhythmic Choice LSSSS SLSSS SSLSS SSSLS SSSSL Mean Cluster 1

\begin{tabular}{|c|c|c|c|c|c|c|}
\hline \multirow[b]{2}{*}{ LSSSS } & & \multirow[b]{2}{*}{18.0} \\
\hline & $56.9^{*}$ & $6.9 \dagger$ & $9.7 \dagger$ & $6.9 \dagger$ & $9.7^{\dagger}$ & \\
\hline SLSSS & $29.2^{*}$ & $70.8^{*}$ & $6.9 \dagger$ & $8.3 \dagger$ & $2.8^{\dagger}$ & 23.6 \\
\hline SSLSS & $5.6 t$ & $1.4 \dagger$ & $56.9^{*}$ & $6.9 \dagger$ & $6.9+$ & 15.5 \\
\hline SSSLS & $1.4 \dagger$ & $8.3 \dagger$ & 13.9 & $68.1^{*}$ & 20.8 & 22.5 \\
\hline SSSSL & $6.9+$ & 12.6 & 12.5 & $9.7 \dagger$ & $59.7^{*}$ & 20.3 \\
\hline$N$ & 72 & 72 & 72 & 72 & 72 & 360 \\
\hline$M S_{\mathrm{e}}$ & 4.7 & 4.7 & 4.7 & 4.7 & 4.7 & 2.1 \\
\hline \multicolumn{7}{|c|}{ Cluster 2} \\
\hline LSSSS & 25.0 & 13.9 & 8.3 & $5.6 \dagger$ & 19.4 & 14.4 \\
\hline SLSSS & 16.7 & 30.6 & 16.7 & 13.9 & 11.1 & 17.8 \\
\hline SSLSS & 25.0 & 27.8 & $36.1^{*}$ & 11.1 & 8.3 & 21.7 \\
\hline SSSLS & 13.9 & 16.7 & $36.1^{*}$ & $50.0^{*}$ & $5.6 \dagger$ & 24.5 \\
\hline SSSSL & 19.4 & 11.1 & $2.8+$ & 19.4 & $55.6^{*}$ & 21.7 \\
\hline$N$ & 36 & 36 & 36 & 36 & 36 & 180 \\
\hline$M S_{e}$ & 6.7 & 6.7 & 6.7 & 6.7 & 6.7 & 3.0 \\
\hline \multicolumn{7}{|c|}{ Cluster 3} \\
\hline LSSSS & 13.9 & 5.6 & 16.7 & 22.2 & 13.9 & 14.5 \\
\hline SLSSS & 30.6 & $36.1^{*}$ & 11.1 & 16.7 & 16.7 & 22.2 \\
\hline SSLSS & 11.1 & 16.7 & 30.6 & 30.6 & 16.7 & 21.1 \\
\hline SSSLS & 27.8 & 22.2 & 27.8 & 19.4 & $33.3^{*}$ & $26.1^{*}$ \\
\hline SSSSL & 16.7 & 19.4 & 13.9 & 11.1 & 19.4 & 16.1 \\
\hline$N$ & 36 & 36 & 36 & 36 & 36 & 180 \\
\hline$M S_{\mathrm{e}}$ & 6.7 & 6.7 & 6.7 & 6.7 & 6.7 & 3.0 \\
\hline \multicolumn{7}{|c|}{ Cluster 4} \\
\hline LSSSS & $38.9^{*}$ & $36.1^{*}$ & 25.0 & 16.7 & 27.8 & $28.9^{*}$ \\
\hline SLSSS & 11.1 & 13.9 & 22.2 & 25.0 & 13.9 & 17.2 \\
\hline SSLSS & 11.1 & 8.3 & 22.2 & 16.7 & 16.7 & 15.0 \\
\hline SSSLS & 13.9 & 16.7 & 19.4 & 27.8 & 30.6 & 21.7 \\
\hline SSSSL & 25.0 & 25.0 & 11.1 & 13.9 & 11.1 & 17.2 \\
\hline$N$ & 36 & 36 & 36 & 36 & 36 & 180 \\
\hline$M S_{\mathrm{e}}$ & 6.7 & 6.7 & 6.7 & 6.7 & 6.7 & 3.0 \\
\hline \multicolumn{7}{|c|}{ Cluster 5} \\
\hline LSSSS & $100.0^{*}$ & $100.0^{*}$ & $100.0^{*}$ & $91.7^{*}$ & $91.7^{*}$ & $97.0^{*}$ \\
\hline SSSSL & & & & $8.3 \dagger$ & $8.3 \dagger$ & $3.0 \dagger$ \\
\hline$N$ & 12 & 12 & 12 & 12 & 12 & 60 \\
\hline$M S_{\mathrm{e}}$ & 11.5 & 11.5 & 11.5 & 11.5 & 11.5 & 5.2 \\
\hline
\end{tabular}

Note-Table entries are percents. *Higher than expected. $\dagger$ Lower than expected $(p<.025)$.

to the accompaniment of $\mathrm{C}_{3}-\mathrm{B}_{3}$ dim- $\mathrm{C} \#_{3}$. Many rhythmic responses were one note away from the rhythm pattern that would start on a chord change; the subjects showed preferences for the rhythms SLSSS, SSSLS, SSLSS, and SSSLS when the chord change supported the rhythms of LSSSS, SSLSS, SSSLS, and SSSSL, respectively. An overall bias was found within Cluster 3 for the rhythm of SSSLS. Members of Cluster 4 showed an overall preference for LSSSS and SSSLS, selecting the former pattern when the chord-change position supported the rhythms LSSSS, SLSSS, or SSLSS, and the latter for when the chord change resulted in the rhythms SSSLS or SSSSL. Cluster 5 consisted of 1 subject who responded with the rhythm LSSSS in all but two occasions.

\section{GENERAL DISCUSSION}

As reported in previous studies on the relative salience of temporal and harmonic contributions to rhythmic structure (Dawe et al., 1993, 1994), the harmonic and temporal features manipulated in this series of experiments were not perceptually equivalent. The data generally support the position taken by Jones (1992) that phenomenal accents are not equally weighted and are in agreement with the emphasis placed on harmonic events by traditional Western music theorists.

The hierarchical solutions and the response profiles for each cluster within the three experiments suggest that the amount of formal musical training may be a predictor for the use and/or nonuse of different rhythmic cues. To test this possibility, four cues were identified as a potential basis for rhythm selection, and the number of responses supporting the use of each was calculated for each subject. These cues included chord-change location, metrical accent (assuming a 6/8 meter in Experiments 1 and 3 and a 3/4 meter in Experiment 2), the onset of the long-duration note (LSSSS), and the ending of the longduration note (SSSSL). A straight-line model was fitted to the data using the least squares approach for each of the four identified cues within each experiment. The number of years of formal musical training was not a reliable predictor for either the use or the nonuse of metrical accents, the onset of the long-duration note, or the termination of the long-duration note; however, it was found to be a reliable predictor for the use of chordchange accents in each of the three experiments [Experiment $1, r=.8699, t(16)=7.06, p<.001$; Experiment 2 , $r=.8258, t(41)=9.376, p<.001$; Experiment $3, r=$ $.7721, t(14)=4.55, p<.001]$.

Overall, musicians had a tendency across the experiments to use a chord change as the basis for reporting a rhythm pattern, whereas nonmusicians were more variable in their use of particular cues, showing a bias to select rhythm patterns consistent with the inferred meter of the experiment. Individual nonmusicians, however, demonstrated a reliable use of specific cues and a bias to report the rhythm pattern consistent with that cue. The result was several clusters of subjects who, in Experiments 1 and 3, showed a bias for either rhythm LSSSS or rhythm SSSLS and, in Experiment 2, showed a bias for the rhythm patterns LSSSS, SSLSS, or SSSSL. The emphasis placed on the harmonic accents by musicians in this study may be indicative of the training and experience they have received in traditional Western music theory, with its heavy emphasis on harmonic functions.

There were some similarities amongst subjects regardless of their training that should be noted. When diatonic chord progressions were used, both musicians and nonmusicians selected rhythm patterns that were defined by accent coincidences. The more accents that coincided, the greater the tendency was for subjects to select the rhythm pattern defined by the coincidence. 
The only exception to the tendency to select on the basis of accent coincidence was in Experiment 3, when the chord progression $\mathrm{C \#}_{3}-\mathrm{B}_{3}$ dim-C $\#_{3}$ was used as the harmonic accompaniment. Under this condition, there was no evidence of an inferred metrical structure or of responses based on coincidence of harmonic and temporal accents. The qualitatively different response profile for this progression requires some explanation. One possible reason for this finding becomes evident when one considers the intervallic information available within an experiment, and from which subjects may determine a tonal center or key (Brown \& Butler, 1981; Butler, 1983, 1989). While there is some ambiguity regarding the tonal center for two of the three trial types in Experiments 1 and 2 (i.e., $\mathrm{G}$ or $\mathrm{C}$ major), there would be no ambiguity if a subject used all the information provided during the experimental session. The tonal center for Experiments 1 and 2 would most likely be $\mathrm{C}$ major. For Experiment 3, the harmonic accompaniment of $\mathrm{C}_{3}-\mathrm{G}_{3}-\mathrm{C}_{3}$ could be interpreted in terms of either $\mathrm{C}$ or $\mathrm{G}$ major, whereas the accompaniment of $\mathrm{G}_{3}-\mathrm{B}_{3} \mathrm{dim}-\mathrm{G}_{3}$ could be interpreted only in terms of $\mathrm{C}$ major. Considering these two trial types, the most likely tonal center would again be $\mathrm{C}$ major. The only harmonic accompaniment in this study that must be interpreted in terms of a modulation between keys is $\mathrm{C}_{3}-\mathrm{B}_{3}$ dim- $\mathrm{C} \#_{3}$.

It may be that subjects interpret an ambiguous rhythmic presentation differently if it is defined by a modulation between keys, as opposed to a progression within a key. If this hypothesis is true, it would account for the similar pattern of responses across harmonic accompaniments and the failure to detect significant differences as a function of harmonic condition in Experiments 1 and 2. Also, not surprisingly, the pattern of responses for the harmonic accompaniments of $C_{3}-G_{3}-C_{3}$ and $G_{3}-B_{3}$ dim- $-G_{3}$ in Experiment 3 were qualitatively similar to the response profiles found in Experiments 1 and 2. This hypothesis may also account for the pattern of responses when the accompaniment was $\mathrm{C}_{3}-\mathrm{B}_{3} \mathrm{dim}-\mathrm{C} \#_{3}$ in Experiment 3.

In conclusion, the results of this study support the notion that the coincidence of accents results in perceptually salient events in music but indicate that simple notions of perceptual accent weight that do not include recognition of structural aspects of a presentation and prior musical training will not be adequate. There were some indications that structural accenting may play a role in the salience of harmonic events. Although any post hoc explanations need further investigation to verify their validity, the results were consistent with the view that subjects inferred a tonal center and changed that reference only in the face of an unlikely or impossible chord within that key. The number of years of formal musical training was found to be a good predictor for the use of chordchange location for perceiving a rhythm pattern. Finally, while there was no direct test of the inferred metrical structure in this study, the pattern of response biases was consistent with the findings of Dawe et al. (1993), suggesting that changes in the composite features of a con- stant harmonic rhythm can result in difference of inferred meter and perceived rhythm.

\section{REFERENCES}

Benjamin, W. (1984). A theory of musical meter. Music Perception, 1 , $355-413$.

BERRY, W. (1976). Structural functions in music. Englewood Cliffs, NJ: Prentice-Hall.

Bharucha, J., \& Krumhansl, C. L. (1983). The representation of harmonic structure in music: Hierarchies of stability as a function of context. Cognition, 13, 63-102.

BoLTZ, M. (1989). Rhythm and "good endings": Effects of temporal structure on tonality judgments. Perception \& Psychophysics, 46, 9-17.

BROWN, H., \& BUTLER, D. (1981). Diatonic trichords as minimal tonal cue-cells. In Theory Only, 5, 37-55.

BUtLer, D. (1983). The initial identification of tonal centers in music. Paper presented to the NATO Conference on the Acquisition of Symbolic Skills, University of Keele, England, July 6-9, 1982. In J. Sloboda \& D. Rogers (Eds.), Acquisition of symbolic skills (pp. 251-261). New York: Plenum.

BUTLER, D. (1989). Describing the perception of tonality in music: A critique of the tonal hierarchy theory and a proposal for a theory of intervallic rivalry. Music Perception, 6, 219-242.

Clarke, E. F. (1985). Structure and expression in rhythmic performance. In P. Howell, I. Cross, \& R. West (Eds.), Musical structure and cognition (pp. 209-236). London: Academic Press.

Clynes, M. (1982). Music, mind, and brain: The neuropsychology of music. New York: Plenum.

Clynes, M. (1986). When time is music. In J. R. Evans \& M. Clynes (Eds.), Rhythm in psychological, linguistic, and musical processes (pp. 164-224). Springfield, IL: Charles C. Thomas.

COOPER, G. W., \& MEYER, L. B. (1960). The rhythmic structure of music. Chicago: University of Chicago Press.

Cuddy, L. L., Cohen, A. J., \& MilleR, J. (1979). Melody recognition: The experimental application of musical rules. Canadian Journal of Psychology, 33, 148-157.

Dawe, L. A., Platt, J. R., \& Racine, R. J. (1993). Harmonic accents in inference of metrical structure and perception of rhythm patterns. Perception \& Psychophysics, 54, 794-807.

Dawe, L. A., PlatT, J, R., \& Racine, R. J. (1994). Inference of metrical structure from perception of iterative pulses within time-spans defined by chord changes. Music Perception, 12, 57-76.

Desain, P. (1992). A (de)composable theory of rhythm perception. Music Perception, 9, 439-454.

Dowling, W. J. (1973). Rhythmic groups and subjective chunks in memory for melodies. Perception \& Psychophysics, 14, 37-40.

EsSENS, P. J., \& POVEL, D. J. (1985). Metrical and nonmetrical representations of temporal patterns. Perception \& Psychophysics, 37, 1-7.

FRAISSE, P. (1963). The psychology of time. New York: Harper \& Row.

Handel, S. (1989). Listening. Cambridge, MA: MIT Press.

JONES, M. R. (1981). Music as a stimulus for psychological motion: Pt. I. Some determinants of expectancies. Psychomusicology, 1, 34-51. JoNEs, M. R. (1982). Music as a stimulus for psychological motion: Pt. II. An expectancy model. Psychomusicology, 2, 1-13.

JONES, M. R. (1984). The patterning of time and its effects on perceiving. In J. Gibbon \& L. Allan (Eds.), Timing and time perception (Annals of the New York Academy of Sciences, Vol. 423, pp. 158167). New York: New York Academy of Sciences.

JONES, M. R. (1986). Attentional rhythmicity in human perception. In J. R. Evans \& M. Clynes (Eds.), Rhythm in psychological, linguistic, and musical processes (pp. 13-40). Springfield, IL: Charles C. Thomas.

JONES, M. R. (1987). Dynamic pattern structure in music: Recent theory and research. Perception \& Psychophysics, 41, 621-634.

JoNEs, M. R. (1990). Musical events and models of musical time. In R. A. Block (Ed.), Cognitive models of psychological time (pp. 207. 240). Hillsdale, NJ: Erlbaum.

JONES, M. R. (1992, February). The structure and function of accent patterns. Paper presented at the Second International Conference on 
Music Perception and Cognition, University of California, Los Angeles.

JONES, M. R., \& Boltz, M. (1989). Dynamic attending and responses to time. Psychological Review, 96, 459-491.

JonES, M. R., BoLtZ, M., \& KIDD, G. (1982). Controlled attending as a function of melodic and temporal context. Perception \& Psychophysics, 32, 211-218.

Krumhansl, C. L. (1985). Perceiving tonal structure in music. American Scientist, 73, 371-378.

KrumhansL, C. L. (1990). Tonal hierarchies and rare intervals in music cognition. Music Perception, 7, 309-324.

Krumhansl, C. L., Bharucha, J. J., \& Kessler, E. J. (1982). Perceived harmonic structure of chords in three related musical keys. Journal of Experimental Psychology: Human Perception \& Performance, 8, 24-36.

Krumhansl, C. L., \& Castellano, M. A. (1983). Dynamic processes in music perception. Memory \& Cognition, 11, 325-334.

KrumhansL, C. L., \& ShePARD, R. N. (1979). Quantification of the hierarchy of tonal functions within a diatonic context. Journal of Experimental Psychology: Human Perception \& Performance, 5, 579-594.

LEE, C. S. (1985). The rhythmic interpretation of simple musical sequences: Towards a perceptual model. In P. Howell, I. Cross, \& R. West (Eds.), Musical structure and cognition (pp. 53-69). London: Academic Press.

LERDAHL, F., \& JACKENDOFF, R. (1983). A generative theory of tonal music. Cambridge, MA: MIT Press.

Longuet-Higgins, H. C., \& LEE, C. S. (1984). The rhythmic interpretation of monophonic music. Music Perception, 10, 424-441.

Palmer, C., \& Krumhansl, C. L. (1987a). Independent temporal and pitch structures in determination of musical phrases. Journal of Experimental Psychology: Human Perception \& Performance, 13, 116-126.

Palmer, C., \& Krumhansl, C. L. (1987b). Pitch and temporal contributions to musical phrase perception: Effects of harmony, performance timing, and familiarity. Perception \& Psychophysics, 41, 505-518.

Palmer, C., \& KRUMhansL, C. L. (1990). Mental representations for musical meter. Journal of Experimental Psychology: Human Perception \& Performance, 16, 728-741.

POVEL, D. J. (1981). Internal representation of simple temporal patterns. Journal of Experimental Psychology: Human Perception \& Performance, 7, 3-18.
Povel, D. J., \& Essens, P. (1985). Perception of temporal patterns. Music Perception, 2, 411-440.

Povel, D. J., \& OKKERMAN, H. (1981). Accents in equitone sequences. Perception \& Psychophysics, 6, 565-572.

SLOBODA, J. A. (1983). The communication of musical metre in piano performance. Quarterly Journal of Experimental Psychology, 35, 377-396.

SMITH, K. C., \& CudDY, L. L. (1989). Effects of metric and harmonic rhythm on the detection of pitch alterations in melodic sequences. Journal of Experimental Psychology: Human Perception \& Performance, 15, 457-471.

Vos, P. (1977). Temporal duration factors in the perception of auditory rhythmic patterns. Scientific Aesthetics, 1, 183-199.

YEstON, M. (1976). The stratification of musical rhythm. New Haven, CT: Yale University Press.

\section{NOTES}

1. Always beginning on the long $(\mathrm{L})$ note may have influenced the perceived organization. Initially, we believed that this would only serve to make the temporal accent perceptually more salient if it had any effect, making selection based on the chord-change location more unlikely. In a pilot study conducted to determine the role of the starting note in the five-note sequence, we found that with 10 repetitions of the sequence, subjects always perceived the rhythm to be LSSSS or SSSSL, in keeping with Gestalt principals of perceptual organization. For more information regarding these earlier investigations, please contact the first author.

2. The onset of every trial's temporal line may have played a role in the preferential selection of LSSSS; however, the first two authors and many subjects in this and other studies have noted how quickly and easily they perceive a particular rhythm pattern independent of how the trial starts or finishes. What the perceived pattern may be is idiosyncratic.

3. This value just reaches significance with a one-tail test, alpha $=$ .05 , if one uses the raw data and not the values reported in the table that have been rounded to the nearest 10th decimal place. Using the tabled values, significance would be reached with a one-tail test, alpha $=.055$.

(Manuscript received May 11, 1993; revision accepted for publication January 2, 1995.) 